\title{
Comparison of integrated traditional Chinese and western medicine therapy on vascular cognitive impairment with no dementia
}

\author{
L.P. Wang', X.Y. Zhang', N. Liu ${ }^{1}$, Z.Z. Ma $^{2}$ and D.S. Fang ${ }^{1}$ \\ ${ }^{1}$ Department of Neurology, Peking University Third Hospital, Beijing, China \\ ${ }^{2}$ Peking University Health Science Centre, Beijing, China \\ Corresponding author: X.Y. Zhang \\ E-mail: rmyangen@163.com
}

Genet. Mol. Res. 14 (2): 4896-4902 (2015)

Received June 7, 2014

Accepted October 30, 2014

Published May 11, 2015

DOI http://dx.doi.org/10.4238/2015.May.11.22

\begin{abstract}
The aim of this study was to investigate the clinical effect of western medicine therapy assisted by Ginkgo biloba tablets (GBT) in patients with vascular cognitive impairment with no dementia (VCIND). Eighty patients with VCIND were randomly divided into two groups: the conventional treatment group (control group) and the combined treatment group. The conventional treatment group was provided with anti-platelet aggregation conventional treatment. In this group, $75 \mathrm{mg}$ aspirin was given three times a day for 3 months, whereas the combined treatment group was given $19.2 \mathrm{mg}$ GBT three times a day for 3 months along with conventional anti-platelet aggregation treatment. Montreal cognitive assessment (MoCA) and transcranial Doppler ultrasonography were used to observe changes in cognitive ability and cerebral blood flow in patients with VCIND before and after treatment in the two groups. After 3 months of treatment, the MoCA scores of execution, attention, abstraction, delayed memory, and orientation were significantly increased in the combined treatment group compared with those before treatment and those in the control group after treatment. In addition, the blood flow velocity of the anterior
\end{abstract}


cerebral artery was significantly increased in the combined treatment group. GBT can improve the therapeutic efficacy, cognitive ability, and cerebral blood flow supply of patients with VCIND.

Key words: Chinese traditional medicine; Western medicine; Vascular cognitive impairment with no dementia; Ginkgo tablet; Cognitive function; Cerebral blood flow

\section{INTRODUCTION}

Disabling chronic diseases among the elderly are a major clinical and public health problem. Dementia, especially Alzheimer's disease, is a prevalent chronic disease currently affecting approximately $24 \%$ of older people ( $\geq 65$ years) in China. The idea that the incidence of dementia in developing countries is lower than that in developed countries is outdated. According to a 2012 World Health Organization survey, 35.6 million individuals worldwide have dementia, and 7.7 million are diagnosed annually. Dementia is a leading cause of agerelated disability and long-term care placement. The herbal product Ginkgo biloba is commonly prescribed to preserve memory; however, its mechanism of action is still unknown. Vascular cognitive impairment (VCI) is the major prodromal period of dementia caused by cerebrovascular disease ( $\mathrm{Li}$ et al., 2011). VCI no dementia (VCIND) is a heterogeneous type of cognitive impairment caused by factors involving the blood vessels. Its cognitive impairment degree ranges between normal and dementia, without reaching the dementia standard (Price et al., 2011). During the pre-dementia stage, memory, together with analysis, judgment, and computation abilities, are significantly impaired, whereas highly familiar skills are usually retained. Cardiovascular dementia is caused by cerebrovascular disease. The remarkable heterogeneity of VCIND is evident in many aspects, such as its risk factors, pathogenesis, pathological processes, clinical characteristics, and treatment reactions (Doruk et al., 2010). Previous studies have shown that VCIND can develop into vascular dementia (VD) (Dong et al., 2010). When VCIND does fully develop into dementia, it can be reversely controlled with medicine intervention (Black, 2007). Therefore, VCIND is the appropriate time for the prevention and control of VD.

Pharmacological research showed that the dry leaves of G. biloba elicit anti-oxidant effects, improve memory, invigorate blood circulation, clear heart channels, induce resuscitation, and so on. These effects are therapeutic in the treatment of ischemic cerebrovascular disease and VD (Xie et al., 1997; Birks and Grimley, 2007).

Two major problems arise in the evaluation of cognitive functions. First, there is no clear normal baseline for the neuropsychological scale used for cognitive appraisals. Second, it is difficult to determine whether cognitive impairment is caused by vessel factors (Dong et al., 2010). In addition, the diagnostic criteria have always been under debate because of their heterogeneity. In this study, the intervening effects of Ginkgo leaves were evaluated in VCIND patients by using the Montreal cognitive assessment (MoCA) rating scale and transcranial Doppler (TCD) ultrasonography. The evaluation was based on aspects of neural psychology and cerebral blood flow. The results of this study should provide a practical foundation and experimental basis for using traditional Chinese medicine as an adjunctive therapy for VCIND. 


\section{MATERIAL AND METHODS}

\section{Diagnosis and standard}

No internationally recognized VCIND diagnostic criteria currently exist. Thus, we referred to related standards and expert advice to establish the following specific standards. The inclusion criteria were as follows: 1) patients were between 60 and 75 years old; 2) the patient stating the condition, whether one's own or that of others, had acquired cognitive impairment for more than 3 months; 3 ) patients were diagnosed with cognitive impairment based on a neuropsychological evaluation; 4) patients showed risk factors of cerebrovascular disease; 5) the existence of a cause and effect relationship between cerebrovascular disease and cognitive impairment; and 6) patients with Hachinski ischemic scores $>7$. The exclusion criteria were as follows: 1) patients who experienced stroke within 3 months; 2) patients with seriously impaired work, social communication, and daily activities; 3 ) patients with cognitive impairment caused by other factors aside from cerebrovascular disease; 4) patients with a history of any type of cardiovascular disease; and 5) patients whose conditions could be diagnosed as dementia.

\section{Subjects}

A total of 80 cerebral infarction patients aged 60 to 75 years (average age $=66.5 \pm 5.6$ years) were treated in our hospital from March 2009 to May 2011, including 46 males and 34 females. Among these patients, 35 had hypertension, 42 had diabetes mellitus, and 33 had hyperlipemia. The patients were randomly divided into two groups of 40: the conventional treatment group (control group) and the combined treatment group.

The control group comprised 23 males and 17 females with an average age of $71.2 \pm 3.5$ years. Some patients had other health conditions: 16 had hypertension, 19 had diabetes mellitus, and 15 had hyperlipemia. The combined treatment group comprised 23 males and 17 females with an average age of $70.8 \pm 3.9$ years. Some patients had other conditions: 19 had hypertension, 23 had diabetes mellitus, and 18 had hyperlipemia. No significant differences in gender, age, the incidence of different complications, cognitive rating score, and TCD detection were found between the two groups $(\mathrm{P}>0.05)$. All subjects were examined by using the MoCA and TCD.

\section{Methods}

The anti-platelet aggregation drug, $75 \mathrm{mg}$ enteric-coated aspirin (Shijiazhuang Pharmaceutical Group, Ouyi Pharmaceutical Industry Ltd., Co.), was given to the conventional treatment group thrice a day for 3 months. Forty milligrams oral G. biloba tablets (GBT; Tanakan EGb761, Tianjinbofu-Yi pu sheng Pharmaceuticals Co., Ltd.) was given to the combined treatment group thrice a day for 3 months. The registered number of the imported drugs was X19990156.

\section{Monitoring indexes}

\section{MoCA}

MoCA contains seven aspects: executive ability, name, memory, attention, language, 
abstraction, delayed memories, and orientation force. Every right answer or operation is equivalent to a score of 1 (memory test does not score). The total score of the scale is 30 , where $\geq 26$ indicates normal cognition and $<26$ indicates cognitive impairment.

\section{TCD evaluation}

Before and after the treatment, a Germany Doppler-Box TCD meter probe (2 MHz electromagnetic pulse) was applied through the temporal windows and pillow window to detect the middle cerebral artery (MCA), anterior cerebral artery (ACA), posterior cerebral artery (PCA), vertebral artery, and basilar artery. The online state of evaluation could display the following in real-time: peak systolic velocity, end-diastolic velocity, average flow velocity, pulsatility index, resistance index, and the systolic peak to diastolic nadir ratio of the blood flow frequency spectrum in two directions. Blood vessels were combined during the outline. According to regional and whole cerebral hemodynamic changes, patients with poor or no transtemporal window displays were eliminated after a comprehensive evaluation of dynamic evolution.

\section{Statistical analysis}

All data were processed with the SPSS13.0 software. Analysis of variance (ANOVA) was used to test measurement data, and the $\chi^{2}$ test was used for count data. $\mathrm{P}<0.05$ was considered to indicate statistical significance.

\section{RESULTS}

\section{Comparison of MoCA score indexes before and after treatment}

Before the treatment, no significant differences in the seven MoCA scores and the total MoCA score $(\mathrm{P}>0.05)$ were found between the conventional and combined treatment groups. In the combined treatment group, significant differences in the scores of five indexes (all except for naming and language $)$ and in the total score were found before and after the treatment $(\mathrm{P}<0.05$ and $\mathrm{P}<0.01$, respectively). After the treatment, significant differences in the scores of executive ability, attention, abstraction, delayed memory, and orientation force, as well as the total MoCA score, were found between the conventional and combined treatment groups $(\mathrm{P}<0.05$; Table 1).

\begin{tabular}{|c|c|c|c|c|}
\hline \multirow[t]{2}{*}{ Index } & \multicolumn{2}{|c|}{ Conventional treatment group } & \multicolumn{2}{|c|}{ Combined treatment group } \\
\hline & Before treatment & After treatment & Before treatment & After treatment \\
\hline Executive ability & $2.25 \pm 1.21$ & $1.97 \pm 1.22$ & $2.49 \pm 0.77$ & $4.02 \pm 0.49^{\Delta *}$ \\
\hline Name & $0.97 \pm 0.56$ & $0.98 \pm 0.45$ & $1.15 \pm 0.93$ & $1.40 \pm 0.56$ \\
\hline Attention & $2.14 \pm 1.05$ & $2.29 \pm 0.73$ & $2.31 \pm 0.41$ & $4.61 \pm 0.42^{\Delta \Delta *}$ \\
\hline Language & $1.51 \pm 0.33$ & $2.02 \pm 0.31$ & $1.42 \pm 0.45$ & $2.22 \pm 0.38$ \\
\hline Abstraction & $0.82 \pm 0.70$ & $0.86 \pm 0.52$ & $0.78 \pm 0.59$ & $1.67 \pm 0.35^{\Delta *}$ \\
\hline Delayed memories & $1.01 \pm 0.84$ & $0.89 \pm 0.71$ & $1.11 \pm 0.56$ & $3.03 \pm 0.37^{\Delta *}$ \\
\hline Orientation force & $4.02 \pm 0.46$ & $3.95 \pm 0.89$ & $3.43 \pm 0.39$ & $5.34 \pm 0.91^{\Delta *}$ \\
\hline Total score & $11.46 \pm 2.98$ & $12.99 \pm 2.81$ & $12.42 \pm 2.66$ & $19.1 \pm 2.94^{\Delta *}$ \\
\hline
\end{tabular}

Data are reported as means $\pm \mathrm{SD} .{ }^{*} \mathrm{P}<0.05$ compared with before treatment. ${ }^{\Delta \Delta} \mathrm{P}<0.01,{ }^{\Delta} \mathrm{P}<0.05$ compared with the control group (after treatment). 


\section{TCD-monitored changes in blood flow of patients in the two groups before and after treatment}

Before the treatment, no significant differences in vascular velocity were found between the conventional and combined treatment groups $(\mathrm{P}>0.05)$. The blood flow velocity of the MCA and ACA in the combined treatment group increased after the treatment. However, no significant differences in blood flow velocity were found before and after the treatment between the conventional and combined treatment groups $(\mathrm{P}<0.05$; Table 2$)$.

\begin{tabular}{|c|c|c|c|c|}
\hline \multirow[t]{2}{*}{ Index } & \multicolumn{2}{|c|}{ Conventional treatment group } & \multicolumn{2}{|c|}{ Combined treatment group } \\
\hline & Before treatment & After treatment & Before treatment & After treatment \\
\hline $\mathrm{MCA}$ & $52.1 \pm 9.7$ & $56.2 \pm 8.9$ & $51.5 \pm 9.4$ & $79.6 \pm 11.2^{\Delta *}$ \\
\hline $\mathrm{ACA}$ & $43.5 \pm 12.2$ & $48.3 \pm 11.1$ & $47.6 \pm 12.8$ & $72.1 \pm 10.3^{\Delta *}$ \\
\hline PCA & $37.6 \pm 5.9$ & $42.3 \pm 7.5$ & $40.2 \pm 6.4$ & $49.8 \pm 9.1$ \\
\hline VA & $42.8 \pm 10.1$ & $45.7 \pm 10.2$ & $42.1 \pm 8.2$ & $52.3 \pm 10.1$ \\
\hline BA & $40.9 \pm 5.8$ & $31.1 \pm 7.7$ & $42.7 \pm 8.8$ & $49.9 \pm 6.5$ \\
\hline
\end{tabular}

\section{DISCUSSION}

VCI has a large set of symptoms ranging from mild cognitive impairment to dementia, which are caused by the risk factors of cerebrovascular disease. Cerebrovascular disease, which may or may not be immediately observable, covers the entire process of VCI from mild to severe, including VCIND, vascular dementia, mixed dementia type, and so on. VCIND is the earliest clinical stage of VCI, and is also likely to be the most common VCI type. VCIND is insidious, and its cognitive impairment degree does not reach the diagnosis standard of dementia (Stephan et al., 2009). Given that VCIND is reversible, the establishment of a simple and effective cognitive function evaluation method is important for the early detection of VCI.

Neuropsychological testing is not only an important tool for VCIND diagnosis, but also for the curative judgment and defection of curative vest. No internationally recognized VCIND neuropsychological testing method is currently available. MoCA is the first recommended VCI neuropsychology screening scale with a consensus among international specialists (Stephan et al., 2009; Dong et al., 2010; Konsztowicz et al., 2011). MoCA is an internationally used screening scale for MCA and VCI that has been verified through evidence-based medicine. This screening scale was improved and established by Nasreddine et al. (2005) based on cognitive item setting and scoring criteria in the Mini-Mental State Examination (MMSE). MoCA places more emphasis on the evaluation of executive function and attention, and is the most important clinical cognitive function tool because of its increased sensitivity compared to the MMSE (Nasreddine et al., 2005). However, its results are influenced by gender, age, and educational level. No statistical differences in gender, age, and educational level of the patients were found in this study, indicating that the present results were unaffected by the aforementioned factors.

Abnormal cerebral blood flow is an important basis for the incidence of VCIND. TCD is a non-invasive cerebrovascular detection tool that directly reflects the hemodynamic 
changes in the intracranial vasculature by determining blood flow velocity and the vascular resistance index (Purandare et al., 2006; Silvestrini et al., 2006; Demarin et al., 2009). TCD is mainly used to detect blood flow states and vascular functions without the interference of social factors, such as education degree, race, and so on. The incidence of abnormal hemodynamics, as determined with TCD, was higher in VCI patients compared with the control group and the cerebral stroke group (Van et al., 2010). A positive correlation was found between MoCA scores and TCD results in this group, indicating that MoCA and TCD can be jointly used for determining mild VCI (Sweet et al., 2011). The TCD results showed that the mean blood flow velocity of the ACA, MCA, and PCA were lower in the combined treatment group than in the conventional treatment group. The blood flow velocity of the ACA and MCA decreased in the cerebral stroke group. The insufficient blood supply of internal carotid circulation may be related to the cognitive impairment of VCIND. The TCD results also showed that the blood flow velocity of the MCA and ACA decreased, indicating insufficient cerebral blood supply in patients.

Flavonoids are the main and effective components of G. biloba leaves. Pharmacological studies revealed that Ginkgo flavonoids and ginkgolides elicit anti-oxidant and anti-radical effects, improve microcirculation, increase cerebral blood flow, improve memory, reduce brain injury, protect smooth muscle cells of cerebral microvessels, improve neural plasticity, reduce the risks of neurodegenerative disease, and so on (Birks et al., 2002; Gertz and Kiefer, 2004; Birks and Grimley, 2007; DeKosky et al., 2008). Considering these advantages, Ginkgo can be used in the early intervention treatment of VCIND patients. This study evaluated the clinical intervention effects of GBT through the combined treatment of GBT and anti-platelet aggregation drug in VCIND patients. Patient scores on executive ability, attention, deferred memory, abstraction, and orientation force all increased significantly after 3 months of combined treatment, indicating that GBT could improve the cognitive function of VCIND patients. The blood flow velocity of the MCA and ACA also increased after taking GBT. This finding indicates that the regional cerebral blood flow in the area where the frontal and temporal lobes are located has a close relationship with the significant increase in cognitive function. Overall, GBT can be used to improve the cognitive ability and cerebral blood flow of patients with VCIND.

\section{REFERENCES}

Birks J and Grimley EJ (2007). Ginkgo biloba for cognitive impairment and dementia. Cochrane Database Syst. Rev. CD003120.

Birks J, Grimley EV and Van Dongen M (2002). Ginkgo biloba for cognitive impairment and dementia. Cochrane Database Syst. Rev. CD003120.

Black SE (2007). Therapeutic issues in vascular dementia: studies, designs and approaches. Can. J. Neurol. Sci. 34 (Suppl 1): S125-S130.

DeKosky ST, Williamson JD, Fitzpatrick AL, Kronmal RA, et al. (2008). Ginkgo biloba for prevention of dementia: a randomized controlled trial. JAMA 300: 2253-2262.

Demarin V, Kes VB, Morović S and Zavoreo I (2009). Evaluation of aging vs dementia by means of neurosonology. $J$. Neurol. Sci. 283: 9-12.

Dong Y, Sharma VK, Chan BP, Venketasubramanian N, et al. (2010). The Montreal Cognitive Assessment (MoCA) is superior to the Mini-Mental State Examination (MMSE) for the detection of vascular cognitive impairment after acute stroke. J. Neurol. Sci. 299: 15-18.

Doruk H, Naharci MI, Bozoglu E, Isik AT, et al. (2010). The relationship between body mass index and incidental mild cognitive impairment, Alzheimer's disease and vascular dementia in elderly. J. Nutr. Health Aging 14: 834-838.

Gertz HJ and Kiefer M (2004). Review about Ginkgo biloba special extract EGb 761 (Ginkgo). Curr. Pharm. Des. 10: 261-264.

Genetics and Molecular Research 14 (2): 4896-4902 (2015)

CFUNPEC-RP www.funpecrp.com.br 
Konsztowicz S, Xie H, Higgins J, Mayo N, et al. (2011). Development of a method for quantifying cognitive ability in the elderly through adaptive test administration. Int. Psychogeriatr. 23: 1116-1123.

Li J, Wang YJ, Zhang M, Xu ZQ, et al. (2011). Vascular risk factors promote conversion from mild cognitive impairment to Alzheimer disease. Neurology 76: 1485-1491.

Nasreddine ZS, Phillips NA, Bedirian V, Charbonneau S, et al. (2005). The Montreal Cognitive Assessment, MoCA: a brief screening tool for mild cognitive impairment. J. Am. Geriatr. Soc. 53: 695-699.

Price CC, Cunningham H, Coronado N, Freedland A, et al. (2011). Clock drawing in the Montreal Cognitive Assessment: recommendations for dementia assessment. Dement. Geriatr. Cogn. Disord. 31: 179-187.

Purandare N, Burns A, Daly KJ, Hardicre J, et al. (2006). Cerebral emboli as a potential cause of Alzheimer's disease and vascular dementia: case-control study. BMJ 332: 1119-1124.

Silvestrini M, Pasqualetti P, Baruffaldi R, Bartolini M, et al. (2006). Cerebrovascular reactivity and cognitive decline in patients with Alzheimer disease. Stroke 37: 1010-1015.

Stephan BC, Matthews FE, Khaw KT, Dufouil C, et al. (2009). Beyond mild cognitive impairment: vascular cognitive impairment, no dementia (VCIND). Alzheimers Res. Ther. 1: 4.

Sweet L, Van Adel M, Metcalf V, Wright L, et al. (2011). The Montreal Cognitive Assessment (MoCA) in geriatric rehabilitation: psychometric properties and association with rehabilitation outcomes. Int. Psychogeriatr. 23: 1582-1591.

Van H, Poommipanit P, Shalaby M, Gevorgyan R, et al. (2010). Sensitivity of transcranial Doppler versus intracardiac echocardiography in the detection of right-to-left shunt. JACC Cardiovasc. Imaging 3: 343-348.

Xie XW, Wang YL, He JG and Han JL (1997). Clinical observation on acute cerebral infarction treated with Ginkgo leaf tablet and 1,6 fibrin degradation products. Integrated Trad. Chin. Western Med. Pract. Crit. Care Med. 6: 21-22. 